\title{
Enzymatic Deposition of Pseudo-Polydopamine Thin Films: A Mild, Versatile and Low-Waste Mussel- Inspired Coating Technology ${ }^{\dagger}$
}

\author{
Maria Laura Alfieri *, Lucia Panzella, Alessandra Napolitano and Marco d'Ischia \\ Department of Chemical Sciences, University of Naples “Federico II”, Naples 80138, Italy; panzella@unina.it \\ (L.P.); alesnapo@unina.it (A.N.); dischia@unina.it (M.d.) \\ * Correspondence: marialaura.alfieri@unina.it \\ + Presented at the 2nd International Online-Conference on Nanomaterials, 15-30 November 2020; Available \\ online: https://iocn2020.sciforum.net/.
}

Published: 15 November 2020

\begin{abstract}
The mimicry of the underwater mussel adhesion strategy for the development of innovative and versatile dip-coating technologies is exemplified by the introduction of polydopamine (PDA) as a highly adhesive biomaterial for surface functionalization and coating, incorporating the key catechol and amine functionalities of byssal proteins. Despite unabated interest and an ever expanding use for various surface functionalization applications, PDA-based technologies suffer from some limitations that have prompted intense studies toward novel musselinspired surface chemistry. Herein, we report the tyrosinase-catalyzed polymerization of tyramine as a mild, versatile and efficient procedure for the development of adhesive PDA-type films ( $\psi$ PDA) that could be obtained at neutral $\mathrm{pH}$ (i.e., 6.8) and at much lower substrate concentration (e.g., $1 \mathrm{mM}$ ) compared to the standard autoxidative PDA coating protocol (typically $10 \mathrm{mM}$ of dopamine). $\psi$-PDA films display structural and physicochemical properties similar to those of PDA films and similar, or even better, antioxidant activity. Confinement or immobilization of tyrosinase at the desired site of functionalization resulted moreover in a highly clean film deposition procedure with complete recovery of unutilized tyramine and no unwanted substrate-consuming autoxidation processes, which limit cost-effectiveness and may interfere with the film deposition procedure.
\end{abstract}

Keywords: mussel-inspired; polydopamine; tyramine; tyrosinase-catalyzed oxidation; coating technology

\section{Introduction}

Deposition of thin films from organic molecules or polymers is an increasingly used strategy to confer specific physical or chemical properties to materials and devices for a variety of biomedical and technological applications. A unique source of inspiration and opportunities in this come from the discovery of the extraordinary wet adhesion properties of polydopamine (PDA), a black insoluble eumelanin-like material produced by oxidative polymerization of dopamine under mild alkaline conditions and inspired to the robust adhesion properties of mussel byssus proteins. Since its first report in 2007 the adhesion properties of polydopamine has been described on all materials and applications have been reported in all fields including e.g., drug delivery, energy storage, molecular detection, bioimaging, catalysis, and nano-interface, to mention only a few [1-3].

Although PDA is currently the reference material for surface functionalization and coating, some limitations have prompted intense studies toward novel mussel-inspired surface chemistry beyond PDA-based technology. They are related to: (a) the intrinsic toxicity of the precursor dopamine; (b) 
the use of an alkaline $\mathrm{pH}$; (c) the need for high dopamine concentrations (10 $\mathrm{mM})$, and (d) difficulties to control film thickness and properties due to the slow kinetics of autoxidation [4,5].

A possible means of bypassing limitations inherent to the autoxidation protocol relies on use of enzymes like tyrosinase, which can be exploited to modulate catecholamine oxidation at $\mathrm{pH}$ values around neutrality [6], leading to uniform films with diverse functionalities [6,7].

Herein, we report the tyrosinase-catalyzed polymerization of tyramine as a mild, versatile and efficient procedure for the development of adhesive PDA-type films ( $\psi$-PDA) that could be obtained at neutral $\mathrm{pH}$ (i.e., 6.8) and at much lower substrate concentration (e.g., $1 \mathrm{mM}$ ) compared to the standard autoxidative PDA coating protocol (typically $10 \mathrm{mM}$ of dopamine) [8]. Finally, the possibility of using tyramine together with confined tyrosinase to achieve site-specific polymerization and/or film deposition is assessed against dopamine. The rationale of the experiments is to prevent the uncontrolled autoxidative deposition of black precipitate, a major drawback of PDA coating technology which may interfere with specific applications [8]. The unifying concept underpinning the new materials is the use of catechol-quinone chemistry as a viable and simple strategy to allow for crosslinking, polymerization and surface adhesion on demand.

\section{Materials and Methods}

All reagents were purchased from Sigma Aldrich and used without further purification. The UV-vis spectra were recorded on a Jasco V-730 Spectrophotometer. Positive reflectron MALDI spectra were recorded on a AB Sciex TOF/TOF 5800 instrument using 2,5-dihydroxybenzoic acid as the matrix.

\subsection{General Procedure for Substrate Coating}

To a $1 \mathrm{mM}$ solution of tyramine or dopamine in $0.05 \mathrm{M}$ sodium carbonate buffer $\mathrm{pH}=6.8$, tyrosinase (1-100 U/mL) was added under vigorous stirring. Substrates (quartz and borosilicate glass coverslips) are dipped into the reaction mixture after complete dissolution of the starting materials and left under stirring up to $24 \mathrm{~h}$, then rinsed with distilled water, sonicated and dried under vacuum.

\subsection{Sodium Alginate Hydrogel}

For preparation of alginate hydrogels, tyrosinase was dissolved in water at $0.1 \%$ or $0.5 \%$, then sodium alginate was added $(2 \% w / w)$. Beads were prepared dropping the freshly prepared gel in a $0.1 \mathrm{M}$ solution of $\mathrm{CaCl}_{2}$ allowing alginate reticulation for few minutes followed by beads filtration. Films were similarly fabricated spreading the gel on a smooth surface followed by dipping in the $\mathrm{CaCl}_{2}$ solution for about $30 \mathrm{~s}$. The beads and films so obtained were washed in distilled water and dipped in tyramine solution $(1 \mathrm{mM})$.

\section{Results}

In the presence of variable amounts of tyrosinase $(20$ and $100 \mathrm{U} / \mathrm{mL})$, the oxidation of tyramine $(1 \mathrm{mM})$ at $\mathrm{pH} 6.8$ resulted after $24 \mathrm{~h}$ in the deposition on various substrates of dark films resembling PDA ( $\psi$-PDA) that could be detected by UV-vis spectrophotometry (Figure 1 ).

Compared to PDA films obtained by similar enzymatic oxidation of $1 \mathrm{mM}$ dopamine with tyrosinase (T-PDA), $\psi$-PDA displayed slower deposition kinetics as evaluated using the Quartz Crystal Microbalance methodology (Figure 2), but similar UV-visible absorption profiles (Figure 1) and lower water-contact angles in the range of $11-28^{\circ}$, denoting higher hydrophilicity. 
A)

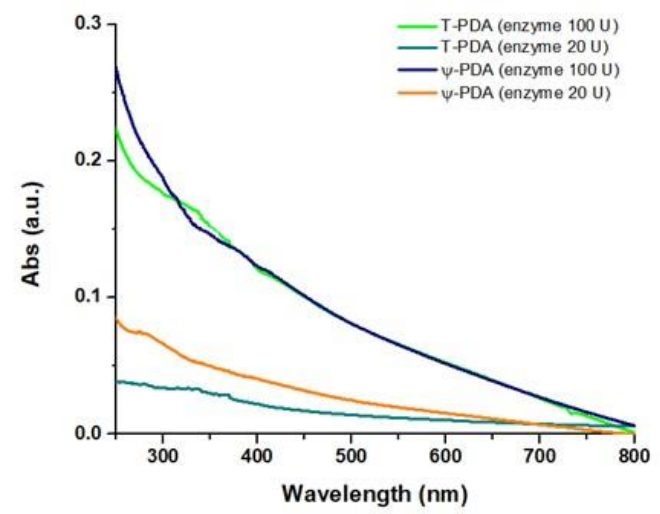

B)

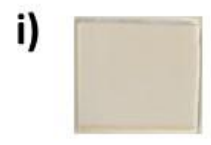

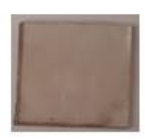
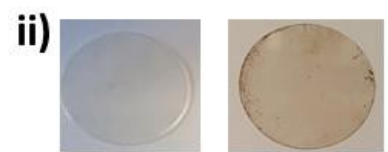

iii)

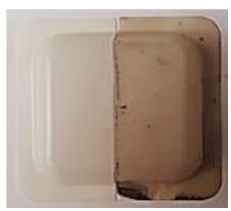

Figure 1. (A) UV-vis spectra of the films obtained from $1 \mathrm{mM}$ tyramine by varying tyrosinase concentration (20 and $100 \mathrm{U} / \mathrm{mL}$ ) compared to PDA films obtained by similar enzymatic oxidation of $1 \mathrm{mM}$ dopamine with tyrosinase. (B) $\psi$-PDA coatings on various materials: (i) quartz; (ii) polycarbonate; and (iii) polystyrene. On the left of each are uncoated materials.

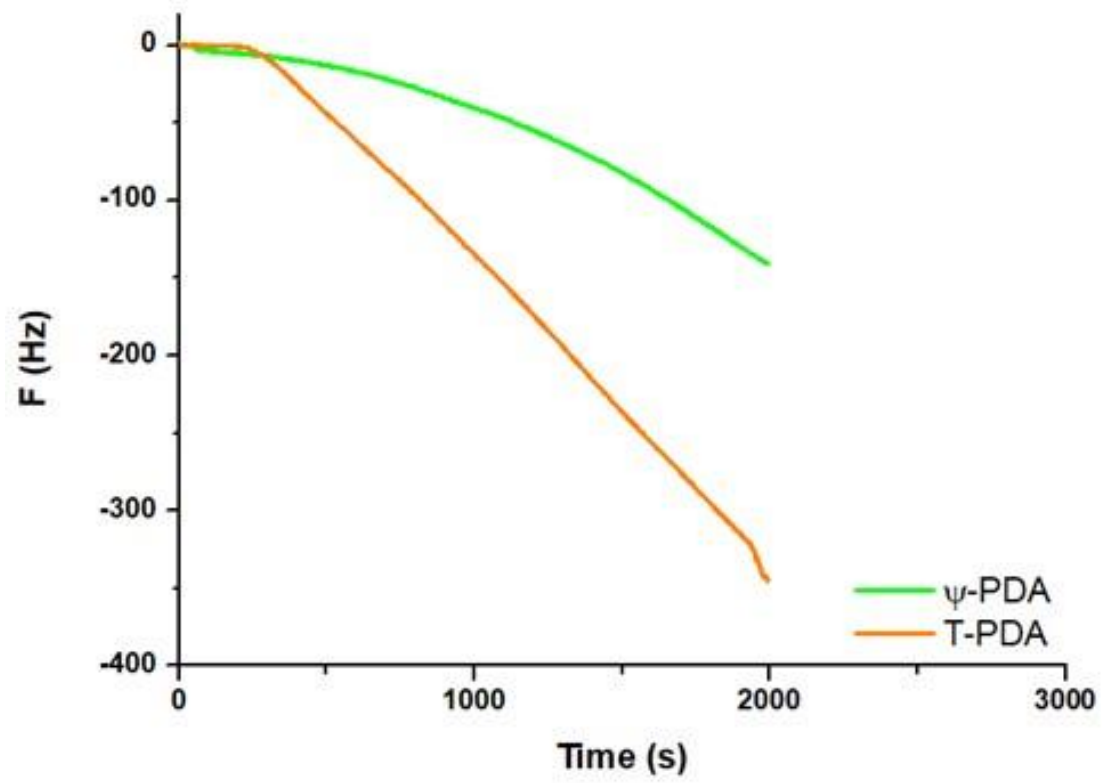

Figure 2. Kinetics of deposition of tyramine or dopamine in the presense of $20 \mathrm{U} / \mathrm{mL}$ of tyrosinase.

Based on these data, the structural properties of the film produced by tyramine-tyrosinase oxidation were investigated by MALDI-MS in comparison with the PDA film. Data in Figure 3 indicate detectable peak clusters at $\mathrm{m} / \mathrm{z}$ ratios up to just below $\mathrm{m} / \mathrm{z} 800$ for PDA but not higher than 700 for tyramine polymer, suggesting for the latter either a lower oxygen content or a different polymerization chemistry. MALDI-MS analysis indicated for $\psi$-PDA a well-defined pattern of peaks compatible with tetrameric structures that had undergone muconic-type cleavage of the corresponding o-quinones, decarboxylation and hydration/dehydration steps. 

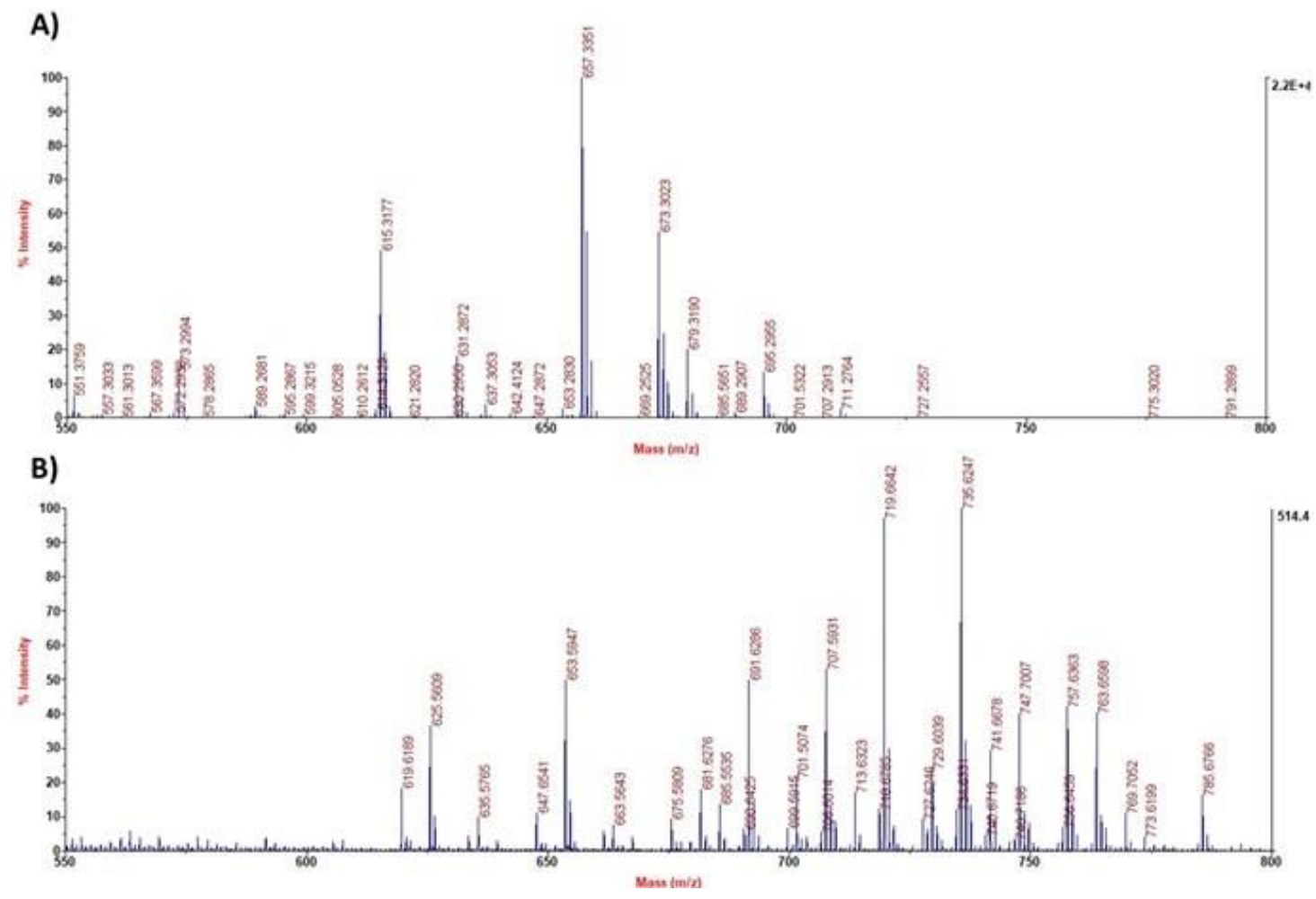

Figure 3. Segmental spectrum of MALDI-ToF ( $m / z$ : 550-800 Da) characterizations of (A) tyramine film in carbonate buffer at $\mathrm{pH}=6.8$ with $20 \mathrm{U} / \mathrm{mL}$ of tyrosinase, (B) PDA film obtained under the same conditions.

Interestingly, exposure to a tyramine solution of tyrosinase-loaded alginate spheres or films deposited on quartz resulted in rapid gel-confined $\psi$-PDA formation with no leakage or darkening of the solution, allowing complete recovery and re-utilization of unreacted tyramine (Figure 4). In contrast, abundant PDA precipitation outside the gel was observed with dopamine under the same conditions. $\psi$-PDA deposition by tyrosinase-catalyzed tyramine oxidation is thus proposed as a controllable and low-waste technology for selective surface functionalization and coating or for clean eumelanin particle production.
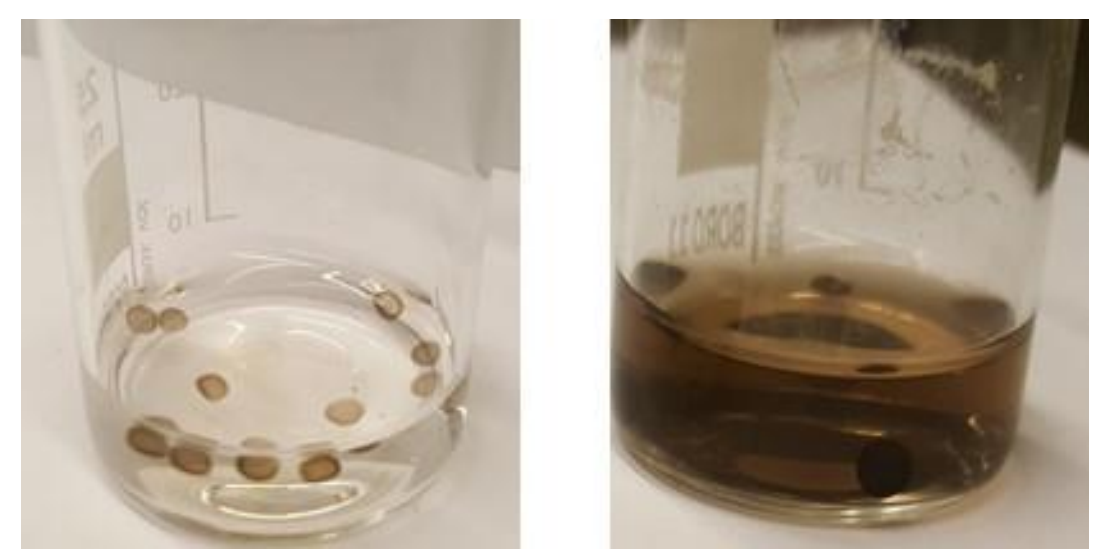

Figure 4 . Tyrosinase $(0.1 \%)$ alginate hydrogel beads incorporating tyrosinase $(0.1 \%)$ and dipped in 1 $\mathrm{mM}$ tyramine (left) or dopamine solution (right) over $4 \mathrm{~h}$.

Acknowledgments: MUR financial support through PRIN project 2017YJMPZN to MdI is acknowledged.

Conflicts of Interest: The authors declare no conflict of interest. 


\section{References}

1. Lee, H.; Dellatore, S.M.; Miller, W.M.; Messersmith, P.B. Mussel-inspired surface chemistry for multifunctional coatings. Science 2007, 318, 426-430.

2. Ryu, J.H.; Messersmith, P.B.; Lee, H. Polydopamine surface chemistry: A decade of discovery. ACS Appl. Mater. Interfaces 2018, 10, 7523-7540.

3. Ball, V. Polydopamine nanomaterials: Recent advances in synthesis methods and applications. Front. Bioeng. Biotechnol. 2018, 6, 109.

4. Lee, B.P.; Messersnith, P.B.; Israelachvili, J.N.; Waite, J.H. Mussel-inspired adhesives and coatings. Annu. Rev. Mater. Res. 2011, 41, 99-132.

5. Ball, V.; Del Frari, D.; Toniazzo, V.; Ruch, D. Kinetics of polydopamine film deposition as a function of $\mathrm{pH}$ and dopamine concentration: Insights in the polydopamine deposition mechanism. J. Colloid Interface Sci. 2012, 386, 366-372.

6. Kim, J.Y.; Kim, W.I.; Youn, W.; Seo, J.; Kim, B.J.; Lee, J.K.; Choi, I.S. Enzymatic film formation of naturederived phenolic amines. Nanoscale 2018, 10, 13351-13355.

7. Zhong, Q.-Z.; Richardson, J.J.; Li, S.; Zhang, W.; Ju, Y.; Li, J.; Pan, S.; Chen, J.; Caruso, F. Expanding the toolbox of metal-phenolic networks via enzyme-mediated assembly. Angew. Chem. Int. Ed. 2019, 59, 17111717.

8. Alfieri, M.L.; Panzella, L.; Youri, A.; Napolitano, A.; Ball, V.; d'Ischia, M. A clean and tunable musselinspired coating technology by enzymatic deposition of pseudo-polydopamine ( $\psi$-PDA) thin films from tyramine Int. J. Mol. Sci. 2020, 21, 4873.

Publisher's Note: MDPI stays neutral with regard to jurisdictional claims in published maps and institutional affiliations.

(C) 2020 by the authors. Submitted for possible open access publication under the terms and conditions of the Creative Commons Attribution (CC BY) license (http://creativecommons.org/licenses/by/4.0/). 